\title{
Combinatorial Identities from the Spectral Theory of Quantum Graphs
}

\author{
Holger Schanz ${ }^{\dagger}$ and Uzy Smilansky ${ }^{\ddagger}$ \\ ${ }^{\dagger}$ Georg-August-Universität and MPI für Strömungsforschung Göttingen, \\ 37073 Göttingen, Germany \\ holger@chaos.gwdg.de \\ $\ddagger$ Department of Physics of Complex Systems, \\ The Weizmann Institute of Science, Rehovot 76100, Israel \\ uzy.smilansky@wicc.weizmann.ac.il \\ Special volume honoring \\ Professor Aviezri Fraenkel
}

Submitted: March 2000; Accepted: April 26, 2000

\begin{abstract}
The purpose of this paper is to present a newly discovered link between three seemingly unrelated subjects - quantum graphs, the theory of random matrix ensembles and combinatorics. We discuss the nature of this connection, and demonstrate it in a special case pertaining to simple graphs, and to the random ensemble of $2 \times 2$ unitary matrices. The corresponding combinatorial problem results in a few identities, which, to the best of our knowledge, were not proven previously.
\end{abstract}

Mathematical Reviews Subject Numbers: 05C38, 90B10

\section{Introduction}

In the present paper we show that some questions arising in the study of spectral correlations for quantum graphs, and in the theory of random matrix ensembles, can be cast as combinatorial problems. This connection will be explained in detail in the next chapter. As a demonstration of this link, we solved in detail a particular system, and the corresponding combinatorial work, resulted in the following identities:

(i) Let $n, q$ be arbitrary integers with $1 \leq q<n$ and

$$
\begin{aligned}
F_{\nu, \nu^{\prime}}(n, q)= & \frac{(n-1) n}{2} \frac{(-1)^{\nu+\nu^{\prime}}}{\nu \nu^{\prime}}\left(\begin{array}{c}
n \\
\nu+\nu^{\prime}
\end{array}\right)^{-1} \\
& \times\left(\begin{array}{c}
q-1 \\
\nu-1
\end{array}\right)\left(\begin{array}{c}
q-1 \\
\nu^{\prime}-1
\end{array}\right)\left(\begin{array}{c}
n-q-1 \\
\nu-1
\end{array}\right)\left(\begin{array}{c}
n-q-1 \\
\nu^{\prime}-1
\end{array}\right) .
\end{aligned}
$$

Then

$$
S(n, q)=\sum_{\nu, \nu^{\prime}=1}^{\min (q, n-q)} F_{\nu, \nu^{\prime}}(n, q)=1
$$


(ii) Let $s, t$ be arbitrary positive integers and

$$
\begin{aligned}
\mathcal{N}(s, t) & =\sum_{\nu=1}^{\min (s, t)}(-1)^{t-\nu}\left(\begin{array}{l}
t \\
\nu
\end{array}\right)\left(\begin{array}{c}
s-1 \\
\nu-1
\end{array}\right) \\
& =(-1)^{s+t}\left(\begin{array}{c}
s+t-1 \\
s
\end{array}\right)^{1 / 2} P_{s+t-1, s}(t),
\end{aligned}
$$

where $P_{N, k}(x)$ are the Kravtchouk polynomials to be defined in Eq. (47). Further, let $x, y$ be complex with $|x|,|y|<1 / \sqrt{2}$. Then we have the generating functions

$$
\begin{aligned}
& G_{1}(x)=\sum_{s, t=1}^{\infty} \mathcal{N}^{2}(s, t) x^{s+t}=\frac{x}{2 x-1}\left(\frac{1}{\sqrt{4 x^{2}+1}}-\frac{1}{1-x}\right), \\
& G_{2}(x)=\sum_{s, t=1}^{\infty} \mathcal{N}(s, t) \mathcal{N}(t, s) x^{s+t}=\frac{1}{2} \frac{4 x^{2}+2 x+1}{(2 x+1) \sqrt{4 x^{2}+1}}-\frac{1}{2}
\end{aligned}
$$

and

$$
g(x, y)=\sum_{s, t=1}^{\infty} \mathcal{N}(s, t) x^{s} y^{t} \frac{x y}{(1+y)(1-x+y-2 x y)} .
$$

(iii) Let $m$ be any positive integer. Then

$$
4 m^{2} \sum_{q=1}^{2 m-1}\left(\frac{\mathcal{N}(2 m-q, q)}{q}\right)^{2}=2^{2 m+1}+(-1)^{m}\left(\begin{array}{c}
2 m \\
m
\end{array}\right)-2
$$

and

$$
(2 m+1)^{2} \sum_{q=1}^{2 m}\left(\frac{\mathcal{N}(2 m+1-q, q)}{q}\right)^{2}=2^{2 m+2}-2(-1)^{m}\left(\begin{array}{c}
2 m \\
m
\end{array}\right)-2 .
$$

(iv) Let $0 \leq q \leq n$, and define

$$
A(n, q)=\frac{1}{\sqrt{2^{n}}} \begin{cases}1 & \text { for } q=0, n \\ (-1)^{q}(n / q) \mathcal{N}(n-q, q) & \text { for } 0<q<n .\end{cases}
$$

Then for any positive integers $0 \leq \kappa \leq \nu$ and an arbitrary integer $n_{0}$,

$$
\lim _{\epsilon \rightarrow 0} \epsilon \sum_{n=n_{0}}^{\infty} \mathrm{e}^{-n \epsilon} \sum_{q=0}^{n} A(n+\nu, q+\kappa) A(n, q)=A(\nu, \kappa) .
$$

We were not able to relate Eqs. (2), (7), (8) to known combinatorial identities including the orthogonality relations for Kravtchouk and Jacobi polynomials. This leads us to believe that these identities are novel. More important is, however, that their derivation establishes a new connection between combinatorics and the theory of random ensembles of unitary matrices. The physical background and motivation is described in a few recent publications $[1,2]$, to which the interested reader is referred. An immediate application of (2) is also given in [2]. Here, we shall provide the minimum background necessary for the understanding of the combinatorial aspects of the problem, and for a self-contained exposition of our results. It will also enable us to propose a conjecture which generalizes the combinatorial approach to random matrix theory for matrices of large dimensions. 


\section{A short Introduction to Quantum Graphs}

We start with a few definitions: Graphs consist of $V$ vertices connected by $B$ bonds (or edges). The valency $v_{i}$ of a vertex $i$ is the number of bonds meeting at that vertex. We shall assume that two vertices can be connected by a single bond at most. We denote the bonds connecting the vertices $i$ and $j$ by $b=[i, j]$. The notation $[i, j]$ will be used whenever we do not need to specify the direction on the bond. Hence $[i, j]=[j, i]$. Directed bonds will be denoted by $d=(i, j)$, and we shall always use the convention that the bond is directed from the first to the second index. If $d=(i, j)$ we use the notation $\hat{d}=(j, i)$. Let $g^{(i)}$ be the set of directed bonds $(i, j)$ which emanate from the vertex $i$, and $\hat{g}^{(i)}$ the set of directed bonds $\left(j^{\prime}, i\right)$ which converge at $i$. The vertices $i$ and $j$ are connected if $g^{(i)} \cap \hat{g}^{(j)} \neq \emptyset$. The bond $d^{\prime}$ is connected to the bond $d$ if there is some vertex $i$ with $d \in g^{(i)}$ and $d^{\prime} \in \hat{g}^{(i)}$.

$d$ and $\hat{d}$ are always connected.

The Schrödinger operator on the graph is defined after the natural metric is assigned to the bonds, and the solutions of the one-dimensional Schrödinger equation on each bond $\left(\mathrm{i} \mathrm{d}_{x}-A\right)^{2} \psi(x)=k^{2} \psi(x)$ is given as a linear combination of counter-propagating waves. $A$ stands here for a magnetic flux. The (complex) amplitudes of the counter propagating waves are denoted by $a_{d}$, where the subscript $d$ stands for the directed bond along which the wave propagates, $d=1, . ., 2 B$. Appropriate boundary conditions at the vertices are imposed, and the spectrum of the Schrödinger operator on the graph is determined as the (infinite, discrete) set of energies $k_{n}^{2}$, for which there exists a non-trivial set of $a_{d}$ which is consistent with the boundary conditions. The condition of consistency can be expressed by the

requirement that

$$
\operatorname{det}(I-S(k))=0
$$

where the bond-scattering matrix $S(k)$ is a unitary operator in the Hilbert space of $2 B$ dimensional vectors of coefficients $a_{d}$. The unitarity of $S(k)$ ensures that the spectrum of the Schrödinger operator is real. The matrix $S(k)$, which is the object of our study is defined as

$$
S_{(i, j),(l, m)}(k)=\delta_{j, l} \mathrm{e}^{\mathrm{i} \phi_{(i, j)}(k)} \sigma_{i, m}^{(j)}(k) .
$$

The matrix elements $S_{d, d^{\prime}}(k)$ vanish if the bonds are not connected. As a consequence, the unitarity of $S$ implies also the unitarity of the $v_{j}$-dimensional vertex-scattering matrices $\sigma_{i, m}^{(j)}$ and vice versa. The phases $\phi_{(i, j)}$, are given in terms of the bond length $L_{[i, j]}$, and the magnetic flux $A_{(i, j)}=-A_{(j, i)}[1]$,

$$
\phi_{(i, j)}(k)=\left(k+A_{(i, j)}\right) L_{[i, j]} .
$$

The two phases pertaining to the same bond $\phi_{d}$ and $\phi_{\hat{d}}$ are equal when $A_{d}=A_{\hat{d}}=0$. In this case $S$ is symmetric and the Schrödinger operator on the graph is invariant under time reversal. Time-reversal symmetry is violated when some magnetic fluxes do not vanish.

$S$ can also be interpreted as a quantum time evolution operator describing the scattering of waves with wave number $k$ between connected bonds. The wave gains the phase 
$\phi_{(i, j)}(k)$ during the propagation along the bond $(i, j)$, while the $\sigma_{i, m}^{(j)}$ describe the scattering at the vertices. In this picture, the unitarity of $S$ guarantees the conservation of probability during the time evolution.

We will avoid unnecessary technical difficulties and consider the matrices $\sigma_{i, m}^{(j)}$ to be $k$ independent constants. One may find explicit expressions for $\sigma_{i, m}^{(j)}$ by requiring besides unitarity that the wave function is continuous at the vertices. The resulting expression is $[1]$

$$
\sigma_{j, j^{\prime}}^{(i)}=\frac{2}{v_{i}}-\delta_{j, j^{\prime}} \quad \text { (Neumann b. c.) } .
$$

Note that back-scattering $\left(j=j^{\prime}\right)$ is singled out both in sign and in magnitude. In all nontrivial cases $\left(v_{i}>2\right)$ the back-scattering amplitude is negative, and the back-scattering probability $\left|\sigma_{j, j}^{(i)}\right|^{2}$ approaches 1 as the valency $v_{i}$ increases.

Finally, a "classical analogue" of the quantum dynamics can be defined as a random walk on the directed bonds, in which the transition probability between bonds $(i, j),(j, l)$ connected at vertex $j$ is $\left|\sigma_{i, l}^{(j)}\right|^{2}$. The resulting classical evolution operator with matrix elements

$$
U_{d, d^{\prime}}=\left|S_{d, d^{\prime}}\right|^{2}
$$

is probability conserving, since unitarity implies $\sum_{i}\left|\sigma_{i, l}^{(j)}\right|^{2}=1$.

\section{Spectral Two-Point Correlations and Periodic-Orbit Sums}

The spectrum of $S$ consists of $2 B$ eigenvalues $\mathrm{e}^{i \theta_{l}}$ which are confined to the unit circle. Their distribution is given in terms of the spectral density

$$
d(\theta) \equiv \sum_{l=1}^{2 B} \delta_{2 \pi}\left(\theta-\theta_{l}\right)=\frac{2 B}{2 \pi}+\frac{1}{2 \pi} \sum_{n=1}^{\infty} s_{n} \mathrm{e}^{-\mathrm{i} \theta n}+\text { c.c. },
$$

where $\delta_{2 \pi}$ denotes the $2 \pi$ periodic delta function. The first

term on the r.h.s. is the average density $\bar{d}=\frac{2 B}{2 \pi}$. The coefficients of the oscillatory part

$$
s_{n}=\operatorname{tr} S^{n}
$$

will play an important rôle in the following. $s_{n}=\operatorname{tr} S^{n}$ is a sum over products of $n$ matrix elements of $S$, and because of (12) the bond indices of each summand describe a connected $n$-cycle ( $\equiv n$-periodic orbit) on the graph

$$
s_{n}=\sum_{p \in \mathcal{P}_{n}} \mathcal{A}_{p} \mathrm{e}^{i \Phi_{p}} .
$$

In (17) $\mathcal{P}_{n}$ denotes the set of all $n$-periodic orbits (PO's) on the graph. Note that for the convenience of presentation we will consider cycles differing only by a cyclic permutation 
as different PO's. The phases $\Phi_{p}=\sum_{j=0}^{n-1} \phi_{d_{j}}$ can be interpreted as the action along the PO $p$. The amplitudes $\mathcal{A}_{p}$ are given by

$$
\mathcal{A}_{p}=\prod_{j=0}^{n-1} S_{d_{j+1}, d_{j}},
$$

where $j$ is understood $\bmod n$. Sometimes it is useful to split the amplitude in its absolute value and a phase $\mathrm{e}^{\mathrm{i} \mu_{p} \pi}$. For example, in the case of Neumann b. c. (14) $\mu_{p}$ is an integer counting the number of back-scatterings along $p$.

In complete analogy to (17) we can represent also the traces of powers of the classical evolution operator

$$
u_{n}=\operatorname{tr} U^{n}
$$

as sums of periodic orbits of the graph.

The two-point correlations in the spectrum of $S(16)$ can be expressed in terms of the average excess probability density $R_{2}(r ; \beta)$ of finding two phases at a distance $r$, where $r$ is measured in units of the mean spacing $\frac{2 \pi}{2 B}$,

$$
\begin{aligned}
R_{2}(r ; \beta) & =\left\langle\frac{1}{2 B} \int_{-\pi}^{+\pi} \mathrm{d} \theta d(\theta) d(\theta-[\pi / B] r)\right\rangle_{\beta}-\frac{B}{\pi} \\
& =\frac{2}{2 \pi} \sum_{n=1}^{\infty} \cos \left(\frac{\pi}{B} n r\right) \frac{1}{2 B}\left\langle\left|s_{n}\right|^{2}\right\rangle_{\beta} .
\end{aligned}
$$

The bond scattering matrix depends parametrically on the phases $\phi_{d}(13)$. We shall define two statistical ensembles for $S$ in the following way. The ensemble for which timereversal symmetry is broken consists of $S$ matrices for which the $\phi_{d}$ are all different, and we consider them as independent variables distributed uniformly on the $2 B$ torus. Invariance under time reversal implies $\phi_{d}=\phi_{\hat{d}}$ and the corresponding ensemble is defined in terms of $B$ independent and uniformly distributed phases. We shall distinguish between these ensembles by the value of the parameter $\beta=\{$ number of independent phases $\} / B$. Expectation values with respect to these measures are denoted in (20) by triangular brackets,

$$
\langle\ldots\rangle_{\beta} \equiv \prod_{d}^{\beta B}\left(\frac{1}{2 \pi} \int_{-\pi}^{+\pi} \mathrm{d} \phi_{d}\right) \ldots .
$$

The Fourier transform of $R_{2}(r ; \beta)$ is the form factor

$$
K(n / 2 B ; \beta)=\frac{1}{2 B}\left\langle\left|s_{n}\right|^{2}\right\rangle_{\beta}
$$

on which our interest will be focussed. If the eigenvalues of the $S$ were statistically independent and uniformly distributed on the unit circle, $K(n / 2 B)=1$ for all $n$. Any deviation of the form factor from unity implies spectral correlations. Using (17) the form factor (22) is expressed as a double sum over PO's

$$
K(n / 2 B ; \beta)=\frac{1}{2 B}\left\langle\left|\sum_{p \in \mathcal{P}_{n}} \mathcal{A}_{p} \mathrm{e}^{\mathrm{i} \Phi_{p}}\right|^{2}\right\rangle_{\beta}
$$




$$
=\frac{1}{2 B} \sum_{p, p^{\prime} \in \mathcal{P}_{n}} \mathcal{A}_{p} \mathcal{A}_{p^{\prime}}^{*}\left\langle\mathrm{e}^{\mathrm{i}\left(\Phi_{p}-\Phi_{p^{\prime}}\right)}\right\rangle_{\beta}
$$

In order to perform the average over all the phases $\phi_{d}$ in (23) we write

$$
\Phi_{p}=\sum_{d} n_{d}^{(p)} \phi_{d}
$$

where $n_{d}^{(p)}$ counts the number of traversals of each directed bond such that $\sum_{d} n_{d}^{(p)}=n$. According to (21) we have

$$
\begin{aligned}
\left\langle\mathrm{e}^{\mathrm{i}\left(n \phi_{d}+n^{\prime} \phi_{d^{\prime}}\right)}\right\rangle_{\beta=1} & =\delta_{n, 0} \delta_{n^{\prime}, 0}, \\
\left\langle\mathrm{e}^{\mathrm{i}\left(n \phi_{d}+\hat{n} \phi_{\hat{d}}\right)}\right\rangle_{\beta=2} & =\delta_{n+\hat{n}, 0} .
\end{aligned}
$$

Thus, the double sum in (23) can be restricted to families of orbits. For $\beta=2$, let $\mathcal{L}$ be the family of isometric PO's which have the same integers $n_{d}^{(\mathcal{L})}$. That is, the family consists of all the PO's which traverse the same directed bonds the same number of times, but not necessarily in the same order. In the case $\beta=1, \mathcal{L}$ consists of all PO's sharing $n_{b}^{(\mathcal{L})} \equiv n_{d}^{(\mathcal{L})}+n_{\hat{d}}^{(\mathcal{L})}$. That is, the family contains all PO's which traverse the same set of undirected bonds the same number of times, irrespective of direction or order. We find

$$
K(n / 2 B ; \beta)=\frac{1}{2 B} \sum_{\mathcal{L} \in \mathcal{F}(\beta)_{n}}\left|\sum_{p \in \mathcal{L}} \mathcal{A}_{p}\right|^{2} .
$$

$\mathcal{F}(\beta)_{n}$ denotes the set of all vectors $\mathcal{L}=\left[n_{d}\right]$ for $\beta=2\left(\mathcal{L}=\left[n_{b}\right]\right.$ for $\left.\beta=1\right)$ of $\beta B$ non-negative integers summing to $n$, for which at least one PO exists. For Neumann b. c. (14), e. g., (27) amounts to counting the PO's in a given set $\mathcal{L}=\left[n_{d}\right]$ taking into account the number of back-scatterings along the orbit. The problem of spectral statistics is now reduced to a counting (combinatorial) problem which is, however, very complicated in general. Even the determination of the number of families $\mathcal{L}$ for a given $n$ is difficult. For $\beta=2$ an obvious necessary condition for the existence of a PO with a given set of bond traversals $\mathcal{L}=\left[n_{d}\right]$ is that at any vertex the number of incoming and outgoing bonds is the same, i. e.

$$
\sum_{d \in g^{(i)}} n_{d}=\sum_{d \in \hat{g}^{(i)}} n_{d} \quad(i=1, \ldots V) .
$$

For $\beta=1$, the analogous condition reads

$$
\sum_{d \in g^{(i)}} n_{d}-\sum_{d \in \hat{g}^{(i)}} n_{d} \bmod 2=0 \quad(i=1, \ldots V),
$$

i. e. the total number of traversals of adjacent bonds should be even at each vertex. However, it is not so easy to formulate a sufficient condition for the existence of a PO given a set of numbers $n_{d}$. In particular one must take care to exclude cases, in which the set of traversed bonds is a union of two or more disconnected groups ("composite orbits"). 
Extensive numerical work [1] revealed that for fully connected graphs $\left(v_{j} \equiv V-1\right)$, and for $V \gg 1$, the form-factor (22) is well reproduced by the predictions of random matrix theory [5] for the Circular Orthogonal Ensemble (COE) $(\beta=1)$ or the Circular Unitary Ensemble (CUE) $(\beta=2)$. This leads us to expect that $(27)$ approaches the corresponding random matrix prediction in the limit $V \rightarrow \infty$. This conjecture is proposed as a challenge to asymptotic combinatorial theory.

\section{The Ring Graph}

In the following we will evaluate explicitly the quantities introduced in the previous section for one of the simplest quantum graphs. It consists of a single vertex on a loop (see fig. 1). There are two directed bonds

$d=1$ and $\hat{d}=2$ with $\phi_{1} \neq \phi_{2}$, i. e. time-reversal symmetry is broken. Since this graph would be trivial for Neumann b.c. the vertex-scattering matrix at the only vertex is chosen as

$$
\sigma(\eta)=\left(\begin{array}{ll}
\cos \eta & \mathrm{i} \sin \eta \\
\mathrm{i} \sin \eta & \cos \eta
\end{array}\right)
$$

with $0 \leq \eta \leq \pi / 2$. The corresponding bond-scattering matrix is

$$
S(\eta)=\left(\begin{array}{ll}
\mathrm{e}^{\phi_{1}} & 0 \\
0 & \mathrm{e}^{\phi_{2}}
\end{array}\right)\left(\begin{array}{ll}
\cos \eta & \mathrm{i} \sin \eta \\
\mathrm{i} \sin \eta & \cos \eta
\end{array}\right) .
$$

We shall compute the form factor for two ensembles. The first is defined by a fixed value of $\eta=\pi / 4$, and the only average is over the phases $\phi_{d}$ according to (25). The second ensemble includes an additional averaging over the parameter $\eta$. We will show that the measure for the integration over $\eta$ can be chosen such that the model yields exactly the CUE form factor for $2 \times 2$ random matrices [5].

\subsection{Periodic Orbit Representation of $u_{n}$}

We will first illustrate our method of deriving combinatorial results from the ring graph in a case where a known identity is obtained. Consider the classical evolution operator $U$ of the ring graph. According to (15) we have

$$
U(\eta)=\left(\begin{array}{cc}
\cos ^{2} \eta & \sin ^{2} \eta \\
\sin ^{2} \eta & \cos ^{2} \eta
\end{array}\right) .
$$

The spectrum of $U$ consists of $\{1, \cos 2 \eta\}$, such that

$$
u_{n}(\eta)=1+\cos ^{n} 2 \eta
$$

We will now show how this result can be obtained from a sum over the periodic orbits of the system, grouped into families of orbits as in (27). In the classical calculation it is actually not necessary to take the families into account, but we would like to stress 
the analogy to the quantum case considered below. The periodic orbit expansion of the classical return probability can easily be obtained from (30) by expanding all matrix products in (19). We find

$$
u_{n}=\sum_{i_{1}=1,2} \ldots \sum_{i_{n}=1,2} \prod_{j=0}^{n-1} U_{i_{j}, i_{j+1}}(\eta)
$$

where $j$ is again taken $\bmod n$. In the following the binary sequence $\left[i_{j}\right]\left(i_{j} \in\{1,2\}\right.$; $j=0, \ldots, n-1)$ is referred to as the code of the orbit. We will now sort the terms in the multiple sum above into families of isometric orbits. In the present case a family is completely specified by the integer $q \equiv q_{1}$ which counts the traversals of the loop 1 , i.e., the number of letters 1 in the code word. Each of these $q$ letters is followed by an uninterrupted sequence of $t_{j} \geq 0$ letters 2 with the restriction that the total number of letters 2 is given by

$$
\sum_{j=1}^{q} t_{j}=n-q
$$

We conclude that each code word in a family $0<q<n$ which starts with $i_{1}=1$ corresponds to an ordered partition of the number $n-q$ into $q$ non-negative integers, while the words starting with $i_{1}=2$ can be viewed as partition of $q$ into $n-q$ summands.

To make this step very clear, consider the following example: All code words of length $n=5$ in the family $q=2$ are 11222, 12122, 12212, 12221 and 22211, 22121, 21221, $22112,21212,21122$. The first four words correspond to the partitions $0+3=1+2=$ $2+1=3+0$ of $n-q=3$ into $q=2$ terms, while the remaining 5 words correspond to $2=0+0+2=0+1+1=1+0+1=0+2+0=1+1+0=2+0+0$.

In the multiple products in (32) a backward scattering along the orbit is expressed by two different consecutive symbols $i_{j} \neq i_{j+1}$ in the code and leads to a factor $\sin ^{2} \eta$, while a forward scattering contributes a factor $\cos ^{2} \eta$. Since the sum is over periodic orbits, the number of back scatterings is always even and we denote it with $2 \nu$. It is then easy to see that $\nu$ corresponds to the number of positive terms in the partitions introduced above, since each such term corresponds to an uninterrupted sequence of symbols 2 enclosed between two symbols 1 or vice versa and thus contributes two back scatterings. For the codes starting with a symbol 1 there are $\left(\begin{array}{l}q \\ \nu\end{array}\right)$ ways to choose the $\nu$ positive terms in the sum of $q$ terms, and there are $\left(\begin{array}{c}n-q-1 \\ \nu-1\end{array}\right)$ ways to decompose $n-q$ into $\nu$ positive summands. After similar reasoning for the codes starting with the symbol 2 we find for the periodic orbit expansion of the classical return probability

$$
\begin{aligned}
u_{n}(\eta)= & 2 \cos ^{2 n} \eta \\
& +\sum_{q=1}^{n-1} \sum_{\nu}\left[\left(\begin{array}{c}
q \\
\nu
\end{array}\right)\left(\begin{array}{c}
n-q-1 \\
\nu-1
\end{array}\right)+\left(\begin{array}{c}
n-q \\
\nu
\end{array}\right)\left(\begin{array}{c}
q-1 \\
\nu-1
\end{array}\right)\right] \sin ^{4 \nu} \eta \cos ^{2 n-4 \nu} \eta \\
= & 2 \cos ^{2 n} \eta+\sum_{q=1}^{n-1} \sum_{\nu} \frac{n}{\nu}\left(\begin{array}{c}
q-1 \\
\nu-1
\end{array}\right)\left(\begin{array}{c}
n-q-1 \\
\nu-1
\end{array}\right) \sin ^{4 \nu} \eta \cos ^{2 n-4 \nu} \eta
\end{aligned}
$$


The summation limits for the variable $\nu$ are implicit since all terms outside vanish due to the properties of the binomial coefficients. From the equivalence between (31) and (34) the combinatorial identity

$$
\sum_{q=1}^{n-1}\left(\begin{array}{c}
q-1 \\
\nu-1
\end{array}\right)\left(\begin{array}{c}
n-q-1 \\
\nu-1
\end{array}\right)=\left(\begin{array}{c}
n-1 \\
2 \nu-1
\end{array}\right)=\frac{2 \nu}{n}\left(\begin{array}{c}
n \\
2 \nu
\end{array}\right) .
$$

could be deduced which indeed reduces (34) to a form

$$
\begin{aligned}
u_{n}(\eta) & =2 \sum_{\nu}\left(\begin{array}{c}
n \\
2 \nu
\end{array}\right) \sin ^{4 \nu} \eta \cos ^{2 n-4 \nu} \eta \\
& =\left(\cos ^{2} \eta+\sin ^{2} \eta\right)^{n}+\left(\cos ^{2} \eta-\sin ^{2} \eta\right)^{n}
\end{aligned}
$$

which is obviously equivalent to (31).

(35) can also be derived by some straight forward variable substitutions from the identity

$$
\sum_{k=l}^{n-m}\left(\begin{array}{l}
k \\
l
\end{array}\right)\left(\begin{array}{c}
n-k \\
m
\end{array}\right)=\left(\begin{array}{c}
n+1 \\
l+m+1
\end{array}\right)
$$

which is found in the literature [8].

\subsection{Quantum Mechanics: Spacing Distribution and Form Factor}

In the following two subsections we derive novel combinatorial identities by applying the reasoning which led to (35) to the quantum evolution operator (29) of the ring graph. We can write the eigenvalues of $S(\eta)$ as $\mathrm{e}^{\mathrm{i}\left(\phi_{1}+\phi_{2}\right) / 2} \mathrm{e}^{ \pm \mathrm{i} \lambda / 2}$ with

$$
\lambda=2 \operatorname{arcos}\left[\cos \eta \cos \left(\frac{\phi_{1}-\phi_{2}}{2}\right)\right]
$$

denoting the difference between the eigenphases. For the two-point correlator we find

$$
\begin{aligned}
R_{2}(r, \eta) & =\left\langle\frac{1}{2} \int_{-\pi}^{+\pi} \mathrm{d} \theta d\left(\theta+\frac{\pi r}{2}\right) d\left(\theta-\frac{\pi r}{2}\right)\right\rangle_{\phi_{1,2}}-\frac{1}{\pi} \\
& =\frac{\delta_{2}(r)-1}{\pi}\left\langle\frac{\delta_{2 \pi}(\pi r+\lambda)+\delta_{2 \pi}(\pi r-\lambda)}{2}\right\rangle_{\phi_{1,2}} \\
& =\frac{\delta_{2}(r)-1}{\pi}+\frac{\sin |\pi r / 2|}{2 \pi} \frac{\Theta\left(\cos ^{2} \eta-\cos ^{2}(\pi r / 2)\right)}{\sqrt{\cos ^{2} \eta-\cos ^{2}(\pi r / 2)}}
\end{aligned}
$$

Here, $\delta_{2}(r)$ is the 2-periodic $\delta$ function. In particular

for equal transmission and reflection probability $(\eta=\pi / 4)$ we have

$$
R_{2}(r, \pi / 4)=\frac{\delta_{2}(r)-1}{\pi}+\frac{1}{2 \pi} \sqrt{\frac{\cos (\pi r)-1}{\cos (\pi r)}} \Theta\left(\frac{1}{2}-|r-1|\right)
$$


and, by a Fourier transformation, we can compute the form factor

$$
\begin{aligned}
K(n, \pi / 4) & =\pi \int_{0}^{2} \mathrm{~d} r \cos (n \pi r) R_{2}(r, \pi / 4) \\
& =1+\frac{(-1)^{m+n}}{2^{2 m+1}}\left(\begin{array}{c}
2 m \\
m
\end{array}\right)-\frac{3}{2} \delta_{n, 0} \\
& \approx 1+\frac{(-1)^{m+n}}{2 \sqrt{\pi n}} \quad(n \gg 1),
\end{aligned}
$$

where $m=[n / 2]$ and [.] stands for the integer part.

Next we consider the ensemble for which transmission and reflection probabilities are uniformly distributed between 0 and 1 . For the parameter $\eta$ this corresponds to the measure $\mathrm{d} \mu(\eta)=2|\cos \eta \sin \eta| \mathrm{d} \eta$. The main reason for this choice is that upon integrating (39) one gets

$$
R_{2}^{(\mathrm{av})}(r)=\frac{\delta_{2}(r)-1}{\pi}+\frac{\sin ^{2}(\pi r / 2)}{\pi}
$$

which coincides with the CUE result for $2 \times 2$ matrices. A Fourier transformation results in

$$
K_{2}(n)=\left\{\begin{array}{ll}
\frac{1}{2} & \text { for } n=1 \\
1 & \text { for } n \geq 2
\end{array} .\right.
$$

The form factors (41), (42) and (44) are displayed in Fig. 1 below.

\subsection{Periodic Orbit Expansion of the Form Factor}

An explicit formulation of (27) for the ring graph is found by labelling and grouping orbits as explained in the derivation of (34). We obtain

$$
\begin{aligned}
K_{2}(n ; \eta)=\cos ^{2 n} \eta+\frac{n^{2}}{2} \sum_{q=1}^{n-1}\left[\sum_{\nu} \frac{(-1)^{\nu}}{\nu}\left(\begin{array}{c}
q-1 \\
\nu-1
\end{array}\right)\left(\begin{array}{c}
n-q-1 \\
\nu-1
\end{array}\right)\right. \\
\\
\left.\times \sin ^{2 \nu} \eta \cos ^{n-2 \nu} \eta\right]^{2}
\end{aligned}
$$

where $q$ denotes the number of traversals of the ring in positive direction

and $2 \nu$ is the number of backward scatterings along the orbit. The inner sum over $\nu$ can be written in terms of Kravtchouk polynomials as

$$
\begin{aligned}
K_{2}(n ; \eta)= & \cos ^{2 n} \eta \\
& +\frac{1}{2} \sum_{q=1}^{n-1}\left(\begin{array}{l}
n-1 \\
n-q
\end{array}\right) \cos ^{2 q} \eta \sin ^{2(n-q)} \eta\left[\frac{n}{q} P_{n-1, n-q}^{\left(\cos ^{2} \eta, \sin ^{2} \eta\right)}(q)\right]^{2},
\end{aligned}
$$

and the Kravtchouk polynomials are defined as in $[3,4]$ by

$$
P_{N, k}^{(u, v)}(x)=\left[\left(\begin{array}{c}
N \\
k
\end{array}\right)(u v)^{k}\right]^{-1 / 2} \sum_{\nu=0}^{k}(-1)^{k-\nu}\left(\begin{array}{l}
x \\
\nu
\end{array}\right)\left(\begin{array}{c}
N-x \\
k-\nu
\end{array}\right) u^{k-\nu} v^{\nu}
$$


for integer $0 \leq k \leq N$ and real positive $u, v$ with $u+v=1$. These functions form a complete system of orthogonal polynomials of integers $x$ with $0 \leq x \leq N$. They have quite diverse applications ranging from the theory of covering codes [6] to the statistical mechanics of polymers [7]. The same functions appear also as a building block in our periodic-orbit theory of Anderson localization on graphs [2]. Unfortunately, we were not able to reduce the above expression any further by using the known sum rules and asymptotic representations for Kravtchouk polynomials. The main obstacle stems from the fact that in our case the three numbers $N, k, x$ in the definition (47) are constrained by $N=k+x-1$.

We will now consider the special case $\eta=\pi / 4$ for which we obtained in the previous subsection the solution (41). The result can be expressed in terms of Kravtchouk polynomials with $u=v=1 / 2$ which is also the most important case for the applications mentioned above. We adopt the common practice to omit the superscript $(u, v)$ in this special case and find

$$
K_{2}(n ; \pi / 4)=\frac{1}{2^{n}}+\frac{1}{2^{n+1}} \sum_{q=1}^{n-1}\left(\begin{array}{l}
n-1 \\
n-q
\end{array}\right)\left[\frac{n}{q} P_{n-1, n-q}(q)\right]^{2} .
$$

It is convenient to introduce at this point the symbol $\mathcal{N}(s, t)$ defined in Eq. (3). It allows to rewrite (48) with the help of some standard transformations of binomial coefficients as

$$
\begin{aligned}
K_{2}(n ; \pi / 4) & =\frac{1}{2^{n}}+\frac{1}{2^{n+1}} \sum_{q=1}^{n-1}\left[\frac{n}{q} \mathcal{N}(q, n-q-1)\right]^{2} \\
& =\frac{1}{2^{n}}+\frac{1}{2^{n+1}} \sum_{q=1}^{n-1}\left[\mathcal{N}(q, n-q)+(-1)^{n} \mathcal{N}(n-q, q)\right]^{2}
\end{aligned}
$$

This expression is displayed in Fig. 1 together with (41) in order to illustrate the equivalence of the two results for the form factor. An independent proof for the equivalence of (41), (48) can be given by comparing the generating functions of $K_{2}(n ; \pi / 4)$ in the two representations [9]. We define

$$
G(x)=\sum_{x=1}^{\infty} K_{2}(n ; \pi / 4)(2 x)^{n} \quad(|x|<1 / 2)
$$

and find from (41)

$$
\begin{aligned}
G(x) & =\frac{2 x}{1-2 x}-\frac{1}{2}+\sum_{m=0}^{\infty} \frac{(-1)^{m}}{2}\left(\begin{array}{c}
2 m \\
m
\end{array}\right) x^{2 m}(1-2 x) \\
& =\frac{1}{2} \frac{1-2 x}{\sqrt{1+4 x^{2}}}-\frac{1}{2} \frac{1-6 x}{1-2 x}
\end{aligned}
$$

On the other hand we have from (49)

$$
G(x)=\frac{x}{1-x}+G_{1}(x)+G_{2}(-x)
$$




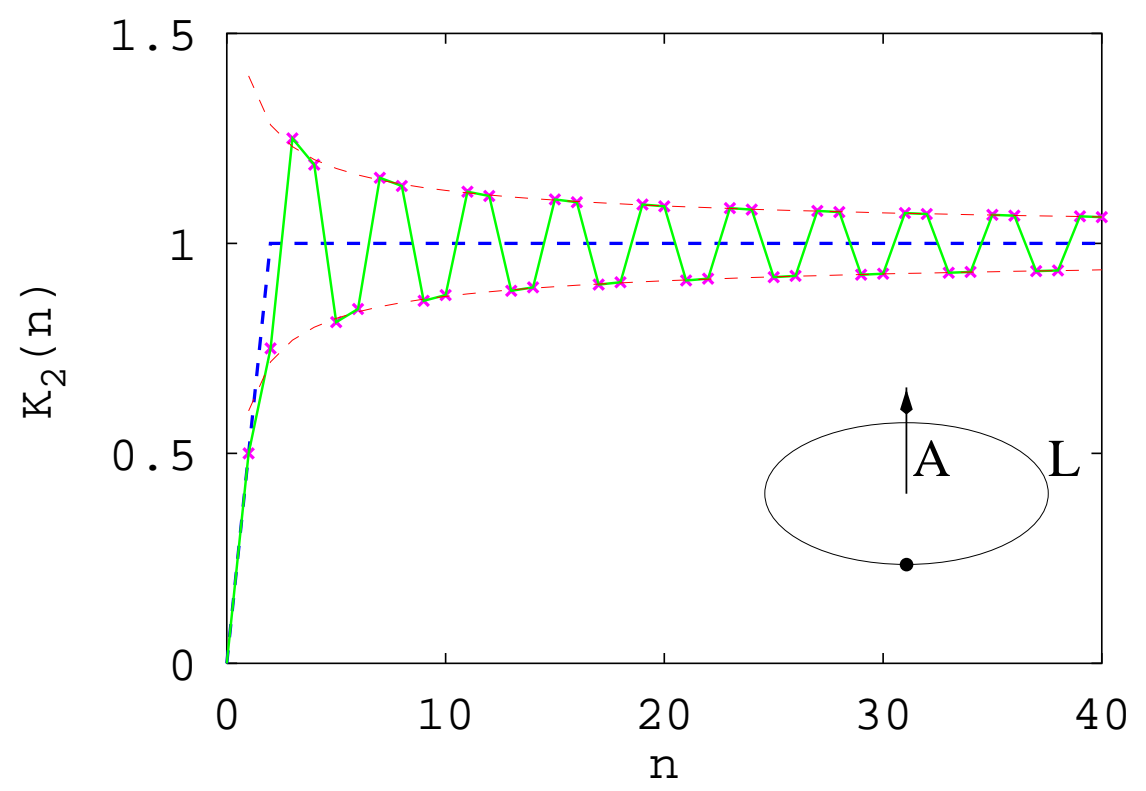

Figure 1: Form factor for the ring graph (see inset). The crosses and the connecting heavy full line show the two equivalent exact results (41) and (48) for $\eta=\pi / 4$. The thin dashed lines represent the approximation (42). The heavy dashed line exhibits the form factor of a CUE ensemble of $2 \times 2$ random matrices (44), which can be obtained by an appropriate averaging over $\eta$ (see text).

with $G_{1,2}(x)$ defined in the introduction. A convenient starting point to obtain the r.h.s. of $(4),(5)$ is the integral representation

$$
\mathcal{N}(s, t)=-\frac{(-1)^{t}}{2 \pi \mathrm{i}} \oint \mathrm{d} z\left(1+z^{-1}\right)^{t}(1-z)^{s-1},
$$

where the contour encircles the origin. With the help of (53) we find

$$
\begin{aligned}
g(x, y) & =\sum_{s, t=1}^{\infty} \mathcal{N}(s, t) x^{s} y^{t} \\
& =-\frac{1}{2 \pi \mathrm{i}} \sum_{s, t=1}^{\infty} \oint \mathrm{d} z \sum_{s, t=1}^{\infty}\left(1+z^{-1}\right)^{t}(1-z)^{s-1} x^{s}(-y)^{t} \\
& =\frac{x y}{2 \pi \mathrm{i}} \sum_{s, t=0}^{\infty} \oint \mathrm{d} z \frac{1}{1-x(1-z)} \frac{1+z}{z+y(1+z)} \\
& =\frac{x y}{(1+y)(1-x+y-2 x y)} \quad(|x|,|y|<1 / \sqrt{2}),
\end{aligned}
$$

which was already stated in (6). The contour $\left|1+z^{-1}\right|=|1-z|=\sqrt{2}$ has been chosen such that both geometric series converge everywhere on it. Now we have

$$
G_{1}\left(x^{2}\right)=\frac{1}{(2 \pi \mathrm{i})^{2}} \oint \frac{\mathrm{d} z \mathrm{~d} z^{\prime}}{z z^{\prime}}
$$




$$
\begin{gathered}
\times \sum_{s, t=1}^{\infty} \sum_{s^{\prime}, t^{\prime}=1}^{\infty} \mathcal{N}(s, t) \mathcal{N}\left(s^{\prime}, t^{\prime}\right)(x z)^{s}(x / z)^{s^{\prime}}\left(x z^{\prime}\right)^{t}\left(x / z^{\prime}\right)^{t^{\prime}} \\
=\frac{x^{4}}{(2 \pi \mathrm{i})^{2}} \oint \mathrm{d} z \mathrm{~d} z^{\prime} \\
\times \frac{1}{\left(1+x z^{\prime}\right)\left(1+x\left[z^{\prime}-z\right]-2 x^{2} z z^{\prime}\right)} \\
\times \frac{z^{\prime}}{\left(z^{\prime}+x\right)\left(z z^{\prime}+x\left[z-z^{\prime}\right]-2 x^{2}\right)}
\end{gathered}
$$

where $|x|<1 / \sqrt{2}$ and the contour for $z, z^{\prime}$ is the unit circle. We perform the double integral using the residua inside the contour and obtain (4) and in complete analogy also (5) such that finally

$$
G(x)=\frac{x}{1-x}+\frac{x}{2 x-1}\left(\frac{1}{\sqrt{4 x^{2}+1}}-\frac{1}{1-x}\right)+\frac{1}{2} \frac{4 x^{2}-2 x+1}{(1-2 x) \sqrt{4 x^{2}+1}}-\frac{1}{2} .
$$

The proof is completed by a straight forward verification of the equivalence between the rational functions (51) and (56).

The identities (7), (8) follow now by separating even and odd powers of $n$ in (41) and (48). In terms of Kravtchouk polynomials these identities can be written as

$$
\sum_{q=1}^{2 m-1}\left(\begin{array}{c}
2 m-1 \\
2 m-q
\end{array}\right)\left[\frac{2 m}{q} P_{2 m-1,2 m-q}(q)\right]^{2}=2^{2 m+1}+(-1)^{m}\left(\begin{array}{c}
2 m \\
m
\end{array}\right)-2
$$

and

$$
\sum_{q=1}^{2 m}\left(\begin{array}{c}
2 m \\
2 m+1-q
\end{array}\right)\left[\frac{2 m+1}{q} P_{2 m, 2 m+1-q}(q)\right]^{2}=2^{2 m+2}-2(-1)^{m}\left(\begin{array}{c}
2 m \\
m
\end{array}\right)-2 .
$$

Finally we will derive the CUE result (44) for the ensemble of graphs defined in the previous subsection starting from the periodic-orbit expansion (45). We find

$$
K_{2}(n)=\int_{0}^{\pi / 2} \mathrm{~d} \mu(\eta) K_{2}(n ; \eta) .
$$

Inserting (45), expanding into a double sum and using

$$
\int_{0}^{\pi / 2} \mathrm{~d} \eta \sin ^{2\left(\nu+\nu^{\prime}\right)+1} \eta \cos ^{2\left(n-\nu-\nu^{\prime}\right)+1} \eta=\frac{1}{2(n+1)}\left(\begin{array}{c}
n \\
\nu+\nu^{\prime}
\end{array}\right)^{-1}
$$

we get

$$
\begin{aligned}
K_{2}(n)= & \frac{1}{n+1}+\frac{n^{2}}{4(n+1)} \sum_{q=1}^{n-1} \sum_{\nu, \nu^{\prime}} \frac{(-1)^{\nu+\nu^{\prime}}}{\nu \nu^{\prime}}\left(\begin{array}{c}
n \\
\nu+\nu^{\prime}
\end{array}\right)^{-1} \\
& \times\left(\begin{array}{c}
q-1 \\
\nu-1
\end{array}\right)\left(\begin{array}{c}
n-q-1 \\
\nu-1
\end{array}\right)\left(\begin{array}{c}
q-1 \\
\nu^{\prime}-1
\end{array}\right)\left(\begin{array}{c}
n-q-1 \\
\nu^{\prime}-1
\end{array}\right) .
\end{aligned}
$$


Comparing this to the equivalent result (44) we were led to the identity (2) involving a multiple sum over binomial coefficients. In this case, an independent computer-generated proof was found [10], which is based on the recursion relation

$$
q^{2} F_{\nu, \nu^{\prime}}(n, q)-(n-q-1)^{2} F_{\nu, \nu^{\prime}}(n, q+1)+(n-1)(n-2 q-1) F_{\nu, \nu^{\prime}}(n+1, q+1)=0 .
$$

This recursion relation was obtained with the help of a Mathematica routine [11], but it can be checked manually in a straight forward calculation. By summing (62) over the indices $\nu, \nu^{\prime}$, the same recursion relation is shown to be valid for $S(n, q)[11,12]$ and the proof is completed by demonstrating the validity of (2) for a few initial values. Having proven (2) we can use it to perform the summation over $\nu, \nu^{\prime}$ in (61) and find

$$
K_{2}(n)=\frac{1}{n+1}+\sum_{q=1}^{n-1} \frac{n}{n^{2}-1}=\frac{1}{n+1}+\frac{n}{n+1}\left(1-\delta_{n, 1}\right),
$$

which is now obviously equivalent to the random-matrix form factor (44).

\subsection{Trace identities}

Let $S$ be an arbitrary unitary matrix with a non degenerate spectrum and $s_{n}=\operatorname{tr} S^{n}$. Then

$$
\lim _{\epsilon \rightarrow 0} \epsilon \sum_{n=n_{0}}^{\infty} s_{n}^{*} s_{n+\nu} \mathrm{e}^{-n \epsilon}=s_{\nu},
$$

for arbitrary integers $n_{0}$ and $\nu[13]$.

We shall now apply this identity to the ring graph with $\eta=\pi / 4$ in order to prove (10). Again, the traces of $S^{n}$ can be expanded in periodic orbits which are grouped into $n+1$ families with equal phases $\Phi(n, q)=q \phi_{1}+(n-q) \phi_{2}(0 \leq q \leq n)$. Thus, one can write

$$
s_{n}(k)=\sum_{q=0}^{n} A(n, q) \mathrm{e}^{\mathrm{i} \Phi(n, q)},
$$

where $A(n, q)$ is the coherent sum of all amplitudes of PO's in the corresponding set. $A(n, q)$ can be expressed in terms of Kravtchouk Polynomials as

$$
A(n, q)=\frac{1}{\sqrt{2^{n}}} \begin{cases}1 & \text { for } \quad q=0 \text { or } n \\
(-1)^{n+q}(n / q)\left(\begin{array}{l}
n-1 \\
n-q
\end{array}\right)^{1 / 2} P_{n-1, n-q}(q) & \text { for } \quad 0<q<n\end{cases}
$$

(compare Eq. (46)). This is equivalent to (9). Substituting (65) into the trace identity (64), we get for arbitrary integers $\nu$ and $n_{0}$

$$
\begin{gathered}
\lim _{\epsilon \rightarrow 0} \epsilon \sum_{n=n_{0}}^{\infty} \mathrm{e}^{-n \epsilon} \sum_{q=0}^{n} \sum_{p=0}^{n+\nu} A(n+\nu, p) A(n, q) \mathrm{e}^{\mathrm{i}\left[(p-q) \phi_{1}+(\nu-(p-q)) \phi_{2}\right]} \\
=\sum_{\kappa=0}^{\nu} A(\nu, \kappa) \mathrm{e}^{\mathrm{i}\left[\kappa \phi_{1}+(\nu-\kappa) \phi_{2}\right]}
\end{gathered}
$$

This is valid for arbitrary phases $\phi_{1}$ and $\phi_{2}$ and therefore the coefficients of the phase factors $\mathrm{e}^{\mathrm{i} \Phi(\nu, \kappa)}$ on both sides are equal. Eq. (10) follows. 


\section{Conclusions}

We have shown how within periodic-orbit theory the problem of finding the form factor (the spectral two-point correlation function) for a quantum graph can be reduced exactly to a well-defined combinatorial problem. Even for the very simple graph model that we considered in the last section the combinatorial problems involved were highly non-trivial. In fact we encountered previously unknown identities which we could not have obtained if it were not for the independent method of computing the form factor directly from the spectrum. However, since the pioneering work documented in [12] the investigation of sums of the type we encountered in this paper is a rapidly developing subject, and it can be expected that finding identities like (2), (7) and (8) will shortly be a matter of computer power.

Numerical simulations in which the form factor was computed for fully connected graphs $\left(v_{i}=V-1 \forall i\right)$ and, e. g., Neumann boundary conditions indicate that the spectral correlations of $S$ match very well Dyson's predictions for the circular ensembles COE or CUE, respectively [1]. The agreement is improved as $V$ increases. We conjecture that this might be rigorously substantiated by asymptotic combinatorial theory. A first step towards this goal was taken in the present paper.

\section{Acknowledgements}

This research was supported by the Minerva Center for Physics of Nonlinear Systems, and by a grant from the Israel Science Foundation. We thank Uri Gavish for introducing us to the combinatorial literature, and Brendan McKay and Herbert Wilf for their interest and support. We are indebted to Gregory Berkolaiko for his idea concerning the proof of

(7) and (8), and to Akalu Tefera for his kind help in obtaining a computer-aided proof of (2).

\section{References}

[1] T. Kottos and U. Smilansky, Phys. Rev. Lett. 79, 4794 (1997); Annals of Physics 274, 76-124 (1999).

[2] H. Schanz and U. Smilansky, Phys. Rev. Lett. 84, 1427-1430 (2000). chaodyn/9904007.

[3] G. Szegö, Orthogonal polynomials, American Mathematical Society Colloquium Publications, Vol. 23, New York (1959).

[4] A. F. Nikiforov, S. K. Suslov, and V. B. Uvarov, Classical Orthogonal Polynomials of a Discrete Variable, Springer Series in Computational Physics, Berlin (1991).

[5] F.J. Dyson. J. Math. Phys. 3 (1962) 140. 
[6] G. Cohen, I. Honkala, S. Litsyn, and A. Lobstein, Covering Codes, North Holland Mathematical Library, Vol. 54, (1997).

[7] K. Schulten, Z. Schulten, and A. Szabo, Physica A 100, 599-614 (1980).

[8] A. P. Prudnikov; J. A. Bryckov; O. I. Maricev, Integrals and Series, Vol. 1, Eq. 4.2.5.39, Gordon and Breach Science Publ., New York (1986). Note that in this edition the relation contains a misprint; the correct form which we provided in the text can easily be proven using [12].

[9] This idea was conveyed to us by G. Berkolaiko.

[10] A. Tefera, private communication.

[11] K. Wegschaider, Computer Generated Proofs of Binomial Multi-Sum Identities, Diploma Thesis, RISC, J. Kepler University, Linz (1997).

[12] M. Petkovšek, H. S. Wilf, and D. Zeilberger $A=B$, AK Peters, Wellesley, Mass. (1996).

[13] U. Smilansky, J. Phys. A 33, 2291-2312 (2000). 